\title{
Nano-scale Compositional Heterogeneity in Pr-doped $\mathrm{CeO}_{2}$
}

\author{
V. Sharma, ${ }^{*}$ K. Eberhardt, ${ }^{*}$ R. Sharma, ** and P.A. Crozier* \\ * School of Materials, Arizona State University, 85287-1504, Tempe, Arizona, USA \\ ** LeRoy Eyring Center for Solid State Science, School of Materials, Arizona State University, \\ Tempe, Arizona, 85287-9506
}

Rare-earth doped cerias have properties which make them suitable anode materials for solid oxide fuel cells (SOFC's) [1]. The redox behavior of the anode material in an SOFC is critical for the efficient conversion of chemical energy (oxidation of hydrogen or hydrocarbons at the anode) to electrical energy. Chemical heterogeneity at the nanoscale is an inherent problem in ceria-zirconia mixed oxides and can modify the performance [2]. In-situ observations have shown that the redox behavior of these oxides depends on the heterogeneity at the atomic level. Here, we report the heterogeneity observed in $\mathrm{Pr}$-doped $\mathrm{CeO}_{2}$ anode material at the nano-scale.

Pure $\mathrm{CeO}_{2}$ and $3 \%$ Pr-doped $\mathrm{CeO}_{2}$ powders were synthesized by drying a $0.05 \mathrm{M}$ solution of $\mathrm{Ce}\left(\mathrm{NO}_{3}\right)_{3} \cdot 6 \mathrm{H}_{2} \mathrm{O}$ and $\operatorname{Pr}\left(\mathrm{NO}_{3}\right)_{3} \cdot 6 \mathrm{H}_{2} \mathrm{O}$, in $1-3 \mathrm{SCFM}$ air flow at $350^{\circ} \mathrm{C}$, using a home-made spray drying system. A part of the sample was calcined at $700^{\circ} \mathrm{C}$ in air. X-ray powder diffraction showed the resulting powder to contain nanoparticles with $7 \mathrm{~nm}$ average diameter and a fluorite structure. High resolution imaging and electron energy-loss spectroscopy were performed using FEI-TECNAI F20 environmental TEM and JEOL 2010F microscopes, operated at 200kV. The contribution from $\mathrm{Pr}$ in doped oxide samples was obtained using the spectra from pure $\mathrm{CeO}_{2}$ for calibration. All spectra were collected, under the same conditions, in diffraction mode with $1 \mathrm{ev} / \mathrm{channel}$ dispersion and a $2 \mathrm{~mm}$ spectrometer aperture size.

HREM images from pre-calcined (Figure 1a) and calcined (Figure 1b) Pr-doped $\mathrm{CeO}_{2}$ samples show that the particle size increases after calcination. EELS spectra collected from individual grains were processed to determine the compositional heterogeneity by measuring relative intensity ratios of $\mathrm{Pr}$ to Ce signals. Several steps were required in the Pr background removal procedure because of the overlap between the $\mathrm{Ce}\left(\right.$ at $890 \mathrm{eV}$ ) and $\operatorname{Pr}\left(\right.$ at $920 \mathrm{eV}$ ) signals. First, an inverse power law ( $\mathrm{AE}^{-\mathrm{r}}$ ) was fitted to the region in front of the Ce edge and extrapolated to remove the background under both the Ce and combine Pr and Ce signals (Fig 2a). The integrated intensity over two regions of the remaining signal, a low energy window $\mathrm{I}_{1}$ (width $10 \mathrm{eV}$ ) involving only the Ce signal and a higher energy window, $\mathrm{I}_{2}$, which contained contributions from both $\mathrm{Pr}$ and $\mathrm{Ce}$ (width $30 \mathrm{eV}$ ) was measured. Similar low and high energy integrals, $\mathrm{I}_{3}$ and $\mathrm{I}_{4}$, were determined for the pure ceria sample (Figure $2 b)$. The Pr signal $I_{P r}$ can then be determine from $I_{P r}=I_{2}-\alpha I_{1}$ where $\alpha=I_{4} / I_{3}$. The best internal consistency was achieved by not including the $\mathrm{Ce}$ white lines in the low energy window. Higher variation in the distribution of Pr was observed in the pre-calcined sample (Figure 3a) compared to the calcined sample (Figure 3(b). It can be concluded that the samples became more homogeneous after calcination. In situ measurements will be used to correlate local composition of the individual particles to the their redox property.

References:

[1] A. Atkinson, S.B., R.J. Gorte, J.T.S. Irvine, A.J. Mcevoy, M. Mogensen, S. C. Singhal, J. Vohs, Nature Materials, 2004. 3: p.17-27. 
[2] Ruigang Wang, P.A. Crozier, Renu Sharma, James B. Adams, Journal of Physical Chemistry B, 2006. 110: p. 18278-18285.

[3] The support from the US Department of Energy (DE-FG02-07ER46442) and the use of TEM at the John M. Cowley Center for High Resolution Microscopy at Arizona State University are gratefully acknowledged.

(a)

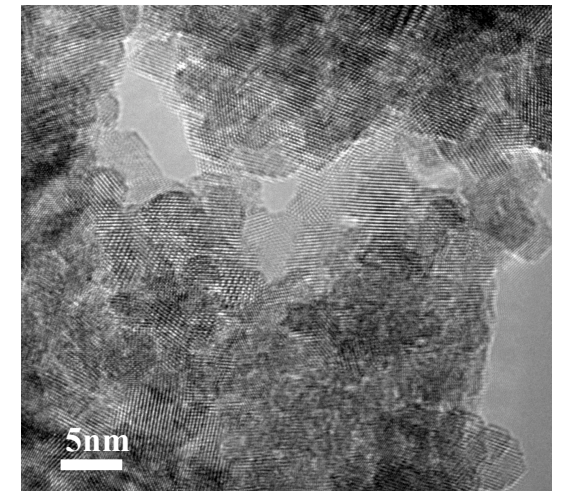

(b)

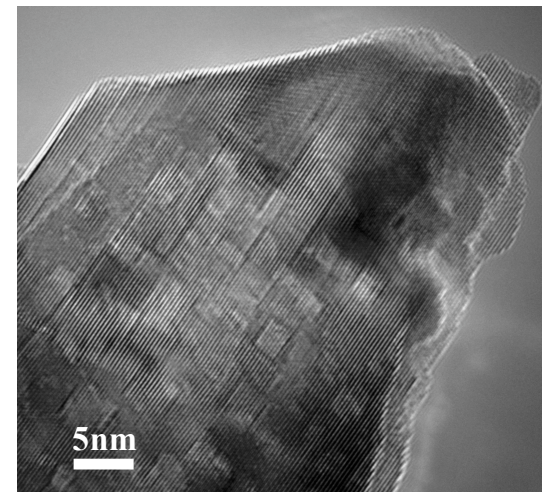

Fig.1. Typical HREM images recorded from (a) pre-calcined and (b) post-calcined $\left(700^{\circ} \mathrm{C}\right) \mathrm{Pr}$-doped $\mathrm{CeO}_{2}$ sample showing an increase in particle size after calicination.

(a)

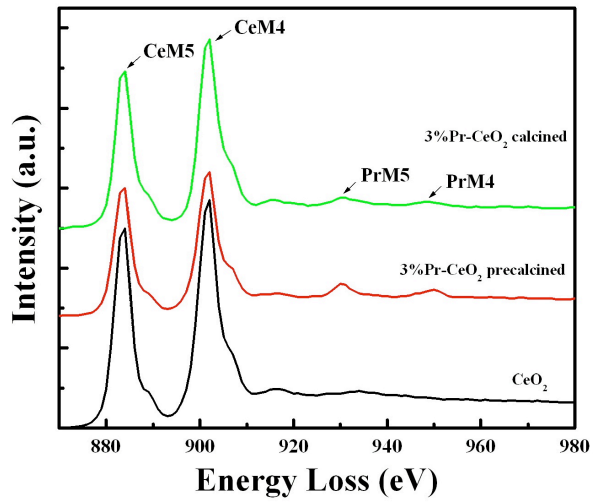

(b)

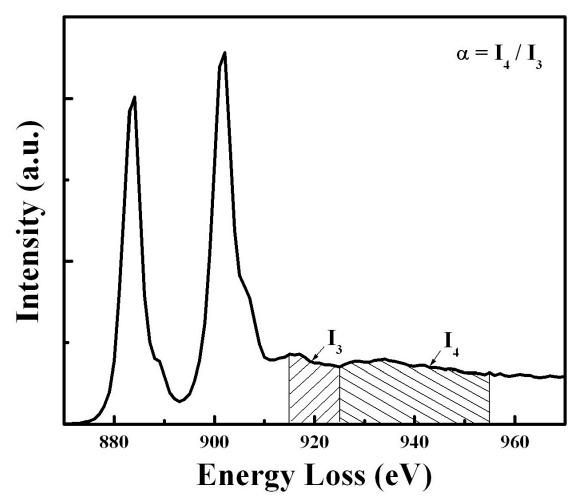

Fig.2. (a) EELS spectra from individual particles of pure $\mathrm{CeO}_{2}$, precalcined and calcined Pr-doped samples respectively, (b) energy windows used to obtain intensity ratios.
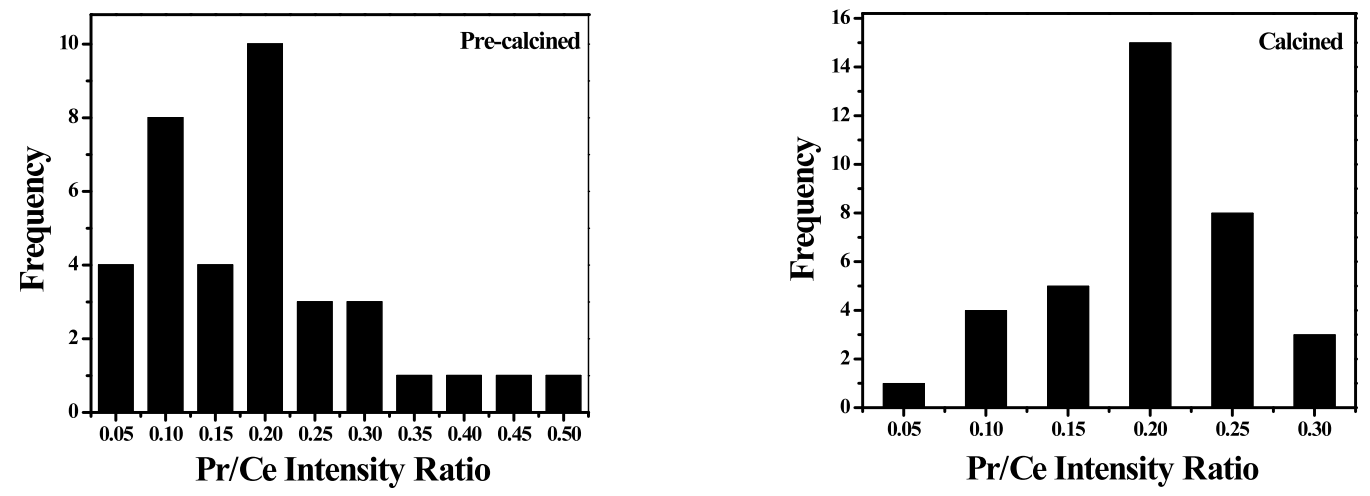

Fig.3. (a) Pr/Ce intensity ratio in individual particles from (a) pre-calcined and (b) post-calcined sample. 